\title{
Research on a new method and system of concentricity measurement of marine propulsion shafting based on structured light technology
}

\author{
Cunjun $\mathrm{Li}^{1, a}$, Xianlei Chen ${ }^{1, b}$ Huadong $\mathrm{Hao}^{1, \mathrm{c}}$ Haolei Shi ${ }^{1, \mathrm{~d}}$ \\ ${ }^{1} 257$ Qiandao Road, Xincheng, Zhoushan city, Zhejiang province, China \\ Zhoushan Institute of Calibration and Testing for Quality and Technology Supervision \\ alicunjun@sina.com bzschenxl@163.com, ${ }^{\mathrm{c}}$ gentlehhd@163.com d181144048@qq.com
}

Keywords: Structured light; Concentricity measurement; Calibration

\begin{abstract}
Based on the structured light technology, a new study method and system of concentricity measurement of marine propulsion shafting is researched in this paper. The system is consisted of the image information acquisition, the structured light calibration and three-dimensional（3D） scanning point cloud data processing. Image information acquisition function is implemented by the hardware part, this part mainly by CCD camera, laser, mechanical rotary table and computer hardware. Image information acquisition is used by the two-dimensional (2D) laser triangulation method. The structured light calibration is based on the internal parameters of the camera and relative external parameters to establish relationship between the image of 2D coordinates and object of the 3D coordinates. The 3D scanning point cloud data processing is included reduction and merging on multi view measurement data. Based on multi view measurement data, an improved ICP algorithm is proposed. This system can provide a new method and a new idea for the concentricity measurement of marine propulsion shafting.
\end{abstract}

\section{Overview}

Marine propulsion shafting includes propeller shaft, intermediate shaft, thrust shaft and connecting piece $^{[1]}$. Due to machine tool spindle rotating unbalance, the force between the tool and the spindle, material strain and other factors in the manufacturing process of shaft system, rotational parts inevitably produce concentricity error, it increases mutual parts wear, vibration and reduce its performance and life. These factors are the adverse impact on safe navigation of the ship. How to accurately concentricity measurement of marine propulsion shafting become a more concern of the maintenance of manufacturing enterprise.

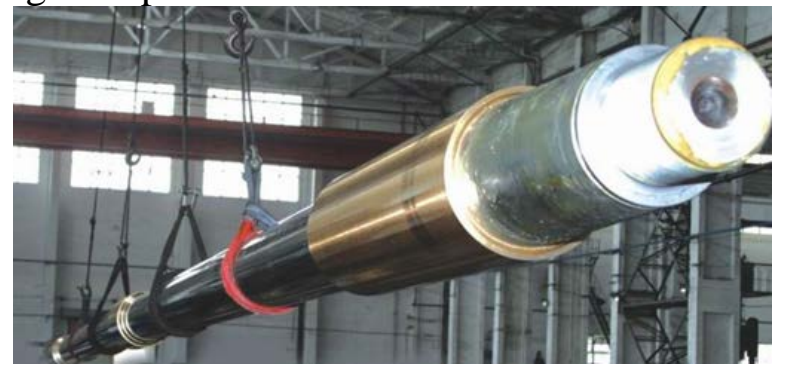

Figure 1 physical map of marine shafting

\section{Research on a new method of concentricity measurement}

Concentricity measurement methods are: indexing head method, laser triangulation method, three coordinate measuring method, computer vision measurement method, two points and three point approximation error measurement method, measurement method based on image processing. Based on the structured light technology, a new study method and the new system of coaxiality measurement of marine propulsion shafting is researched. 
At present, with advantage of the high-speed, high-precision, stereo imaging and non contact, 3D scanning technology gradually emerge in all walks of life. In ship industry, 3D scanning technology has been applied to the detection of ship parts, ship construction and design error ratio, ship structure modeling and other related issues. 3D scanning technology has become a popular technology in the ship detection. The structured light measurement technology has been widely used in the reverse engineering, quality inspection, digital artifacts, virtual reality and other fields.

As shown in Figure $2^{[2,3]}$, the line structured light system is generally composed of a light projector, a camera and a scanning device. Laser projected the light plane to the object surface through a cylindrical lens. By the depth information modulated of the object surface, the camera collects the deformed light stripe image and calculates the coordinates of the center of the light stripe center. According to the positional of triangle relationship between the projector and the camera, get 3D information form deformation stripes on the measured surface. The calibration of the center of light stripe, the position of the light plane and the position of the camera directly affect the accuracy of the system.

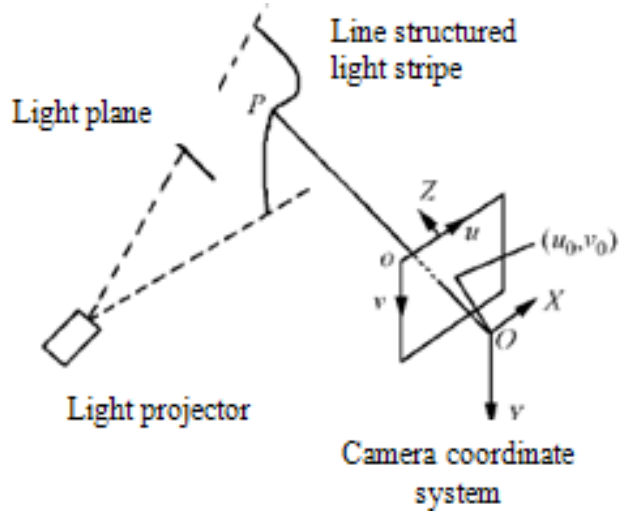

Figure 2 Schematic diagram of line structure light measurement

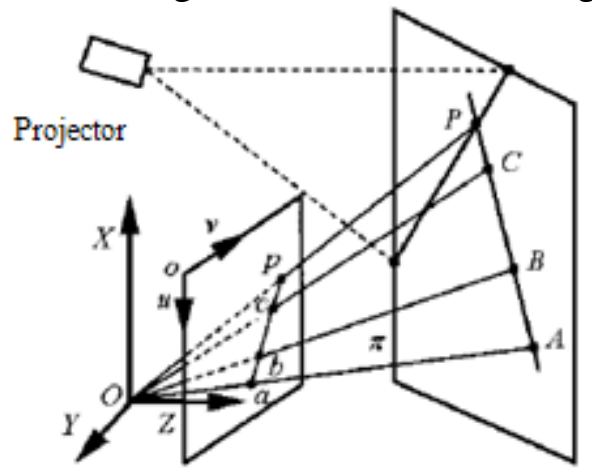

Figure 3 Schematic diagram of light plane calibration

In image processing, first the light of the position initially identified by threshold method; Second get the normal direction of the light by the light intensity gradient information; Finally get light strip center coordinates in the normal direction of light intensity on Gaussian fitting. For example, traditional light plane calibration method with wire drawing method, serrated target method, coplanar reference object method and based on cross ratio invariant 3D target method.

\section{System overview}

The system consists of acquisition of the image information, the structure light calibration and 3D point cloud data processing.

(1)Design of system hardware

The system consists of acquisition of the image information, the structure light calibration and 3D point cloud data processing. Image information acquisition function is implemented by the hardware part, this part mainly by CCD camera, laser, mechanical and computer hardware. Image acquisition is used a 2D laser triangulation method. 2D laser triangulation method is based on stereo vision effect of 
the human eye. Two CCD cameras receiver object, each CCD camera and laser beam constitute a ranging sensor based on triangulation. As shown in Figure 4, the design of this paper is based on the dual triangulation laser ranging sensor, consisting of a line laser generator and two CCD

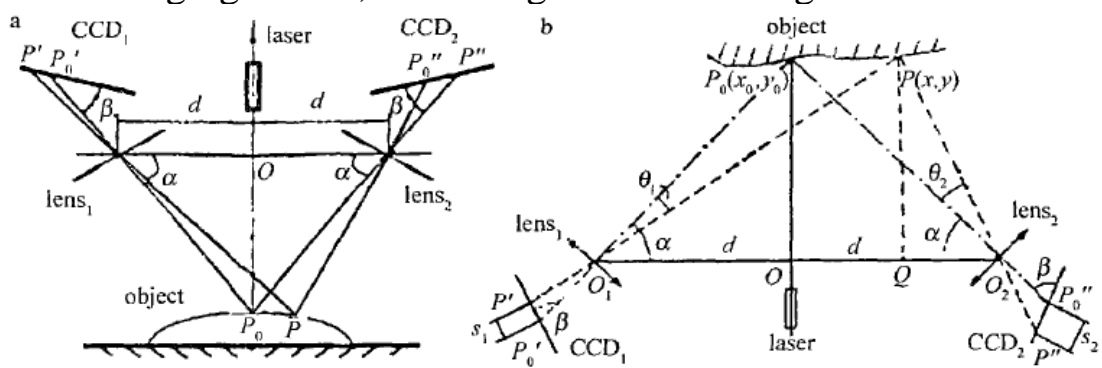

Figure 4 Schematic diagram of 2D laser triangulation

cameras. The principle is as follows:

According to the law of cosines are:

$O_{1} P^{\prime}=\sqrt{s_{1}^{2}+f^{2}-2 s_{1} \cdot f \cdot \cos \beta}$.

$\mathrm{O}_{2} P^{\prime \prime}=\sqrt{s_{2}^{2}+f^{2}-2 s_{2} \cdot f \cdot \cos \beta}$.

According to the law of sine are:

$\theta_{1}=\arg \sin \left(s_{1} \bullet \sin \beta / O_{1} P^{\prime}\right)$.

$\theta_{2}=\arg \sin \left(s_{2} \bullet \sin \beta / O_{2} P^{\prime \prime}\right)$.

$P O_{1}=\frac{2 d \cdot \sin \left(\alpha+\theta_{2}\right)}{\sin \left(\pi-2 \alpha+\theta_{1}-\theta_{2}\right)}$.

$P O_{2}=\frac{2 d \cdot \sin \left(\alpha-\theta_{1}\right)}{\sin \left(\pi-2 \alpha+\theta_{1}-\theta_{2}\right)}$.

According to the relationship of triangular, the 2D coordinates of the point $\mathrm{P}$ can be obtained:

$$
\begin{aligned}
& x=2 d\left[1-\frac{\sin \left(\alpha-\theta_{1}\right) \cdot \cos \left(\alpha+\theta_{2}\right)}{\sin \left(\pi-2 \alpha+\theta_{1}-\theta_{2}\right)}\right] . \\
& y=\frac{2 d \cdot \sin \left(\alpha-\theta_{1}\right) \cdot \sin \left(\alpha+\theta_{2}\right)}{\sin \left(\pi-2 \alpha+\theta_{1}-\theta_{2}\right)} .
\end{aligned}
$$

In the equation, $\mathrm{B}$ is the angle between CCD camera image plane and the corresponding camera optic axis of lens; $A$ is the angle between the laser beam and the optical axis of the lens; $F$ is the focal length of the lens; $2 d$ is the distance between the two camera center of lens; S1 and s2 respectively as the distance from the center of the two face reading; Adopt dual triangle method can get the 2D coordinates of points on the object, the 3D coordinate information can be achieved through the scanning movement of the laser line.

(2)Design of structured light calibration system

In the research of stereo vision, using two cameras to simulate human eyes, the two-dimensional images of the object from different angles by cameras is obtained ${ }^{[4]}$. The structure light calibration is based on the internal parameters of the camera and relative external parameter, established relationship between the image of $2 \mathrm{D}$ coordinates and objects of the $3 \mathrm{D}$ coordinates. Therefore, the camera calibration is a prerequisite for the realization of binocular stereo vision. With the mechanical 
scanning motion, the coordinates of the surface of the object can be obtained. Coordinate system as follows: Cartesian coordinate system of $O_{P}-u v$ is defined on the digital image; $(u, v)$ is coordinates of each pixel represented the number of columns and rows of pixels in the image array; $(u, v)$ is the coordinates of image point in the digital image coordinate system in pixels.

$O_{i}-x y$ is the coordinate of $2 \mathrm{D}$ image plane expressed in physical units. The $\mathrm{X}$ and $\mathrm{Y}$ axes are parallel to the $\mathrm{Z}$ axis and the $\mathrm{U}$ axis. The origin is the intersection of the camera optical axis and the the image plane. Set up camera coordinate system $O_{c}-x_{c} y_{c} z_{c}$. The $x_{c}$ and $y_{c}$ axes are parallel to the $x$ axis and the $y$ axis of the image plane. $Z_{c}$ axis is the optical axis of the camera, which is perpendicular to the image plane. Structured light plane parallel to $O_{c}$ and angle of the optical axis of camera is $\alpha$. Take the origin of the world coordinate system $O_{w}-x_{w} y_{w} z_{w} O_{w}$ located at the intersection of camera and structured light plane. The $x_{w}$ and $y_{w}$ axes are parallel to the $x_{c}$ axis and the $y_{c}$ axis of the camera coordinate system. $Z_{w}$ coincide with $Z_{c}$ but in the opposite direction. The distance of $O_{w}$ and $O_{c}$ is the $l$. According to the cameraDLT model:

$$
\begin{aligned}
& Z_{c}[u v l]^{\mathrm{T}}=w L\left[X_{w} Y_{w} Z_{w}\right]^{\mathrm{T}} . \\
& L=\left[\begin{array}{l}
L_{1} L_{2} L_{3} L_{4} \\
L_{5} L_{6} L_{7} L_{8} \\
L_{9} L_{10} L_{11} 1
\end{array}\right] . \\
& \left\{\begin{array}{l}
u=\frac{L_{1} X_{w}+L_{2} Y_{w}+L_{3} Z_{w}+L_{4}}{L_{9} X_{w}+L_{10} Y_{w}+L_{11} Z_{w}+1} \\
v=\frac{L_{5} X_{w}+L_{6} Y_{w}+L_{7} Z_{w}+L_{8}}{L_{9} X_{w}+L_{10} Y_{w}+L_{11} Z_{w}+1}
\end{array}\right.
\end{aligned}
$$

Equation (11) is the DLT model of the direct linear transformation of the camera. In the structure optical rotation scanning system, the world coordinate is often used in the column coordinate system. Set up column coordinate system $O_{r}-r h \varphi . O_{r}$ coincide with $O_{w}$. $h$ coincide with $y_{w}$. The direction of $Z_{w}$ is $\varphi=0$.

Constrained by the structured light plane, the points of structured light are:

$Z_{c} P=w A C$.

In the equation, $C=[r h l]^{\mathrm{T}} ; P=[u v l]^{\mathrm{T}}$; $\mathrm{A}$ is $3 \times 3$ matrix. The equation (12) is a DLT model based on the structured light plane constraint.

According to the DLT model based on plane constraint, in the column coordinate system, on the left camera are:

$$
\begin{aligned}
& w_{\mathrm{L}} A_{\mathrm{L}} C=Z_{\mathrm{cL}} P_{\mathrm{L}} . \\
& C=Z_{\mathrm{cL}} A_{\mathrm{L}}^{-1} P_{\mathrm{L}} / w_{\mathrm{L}} .
\end{aligned}
$$

On the right camera are:

$$
\begin{aligned}
& w_{\mathrm{R}} A_{\mathrm{R}} C=Z_{\mathrm{cR}} P_{\mathrm{R}} . \\
& P_{R}=Z_{\mathrm{cL}} w_{R} A_{R} A_{\mathrm{L}}^{-1} P_{\mathrm{L}} /\left(Z_{\mathrm{cR}} w_{\mathrm{L}}\right) .
\end{aligned}
$$




$$
P_{R}=k\left[\begin{array}{l}
d_{11} d_{12} d_{13} \\
d_{21} d_{22} d_{23} \\
d_{31} d_{32} 1
\end{array}\right] P_{\mathrm{L}}=k D P_{\mathrm{L}}
$$

$D$ is called the two camera mapping matrix. Through the calibration, it can be calculated from the image coordinates of the left camera at a position in the right camera image. That is:

$$
\left\{\begin{array}{l}
u_{\mathrm{R}}=\frac{d_{11} u_{\mathrm{L}}+d_{12} v_{\mathrm{L}}+d_{13}}{d_{31} u_{\mathrm{L}}+d_{32} v_{\mathrm{L}}+1} \\
v_{\mathrm{R}}=\frac{d_{21} u_{\mathrm{L}}+d_{22} v_{\mathrm{L}}+d_{23}}{d_{31} u_{\mathrm{L}}+d_{32} v_{\mathrm{L}}+1}
\end{array} .\right.
$$

Two camera field of view cannot be completely coincident, the above derivation is only two camera field of view is set up. Equation (18) is a reflection of the corresponding relationship between the two camera structured light images in a dual camera structured light system.

According to the equation (16) and equation (17), a point $i$ on the plane of the structured light is located in the common vision of the two cameras, two equations can be obtained:

$$
\begin{aligned}
& {\left[\begin{array}{ll}
u_{\mathrm{L} i} & 0 \\
v_{\mathrm{L} i} & 0 \\
1 & 0 \\
0 & u_{\mathrm{L} i} \\
0 & v_{\mathrm{L} i} \\
0 & 1 \\
-u_{\mathrm{R} i} u_{\mathrm{L} i} & -v_{\mathrm{R} i} u_{\mathrm{L} i} \\
-u_{\mathrm{R} i} v_{\mathrm{L} i} & -v_{\mathrm{R} i} v_{\mathrm{L} i}
\end{array}\right]^{\mathrm{T}} d=\left[\begin{array}{l}
u_{\mathrm{R} i} \\
v_{\mathrm{R} i}
\end{array}\right]} \\
& d=\left[d_{11}, d_{12}, d_{13}, d_{21}, d_{22}, d_{23}, d_{31}, d_{32}\right]^{\mathrm{T}} .
\end{aligned}
$$

From three or more known points can be calculated $d$ using the least squares method to obtain a transformation matrix $D$. Using this method to calibrate the dual camera in the structured light system, it is not need to know the space coordinates of the calibration point. In addition, the two cameras respectively can be calibrated, and get their respective parameters matrix, and then solved the relationship between them.

(3)Process of the 3D scanning point cloud data

It is necessary to carry out the preprocessing of point cloud data before surface reconstruction, including reduction and merging on multi view measurement data. A combination of curvature change and space reduction is proposed to simplify the point cloud data. This method not only effectively reduces the number of points cloud, but also maintains the geometrical features of the object more accurately, and can improve the speed of the subsequent surface reconstruction. The space division of the point cloud is made by using the 3D grid method. This method avoids the repeated calculation of the distance, improves the searching speed of the points, and provides a good basis for the following surface reconstruction and modeling. In multi view measurement data, proposed an improved Iterative Closest Points (ICP) algorithm. By calculating the measured object surface feature points, this method using curvature of similar point as the initial matching point pairs, using the K-D tree to search for the nearest point accelerated. The method is applied ICP algorithm to solve the feature points to match the least squares objective function, using K-D tree to construct a reasonable data structure for point cloud data. 


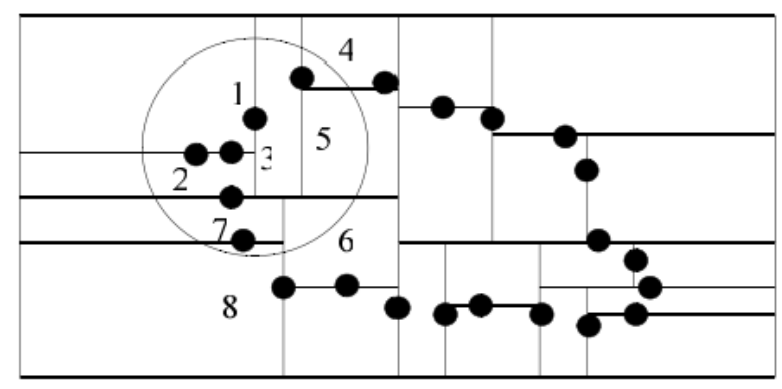

Figure 5 Schematic diagram of tree structure

As shown in Figure 5, black dots represent the input points; Circle represents the scope of inquiry; A digital representation of the query sequence. Each node is a symbol of a rectangular area and the corresponding to the axis of the coordinate, corresponding node of the partition line and its depth should also be. Determine the neighborhood of each point in the point cloud, and then look for the closest points by K-D tree. Reconstruction of radial basis functions resulting surface is implicit surfaces, the application should be implicit surfaces extracted into the grid. The surface following algorithm is used to extract and reduce the computational complexity and improve the solution speed. The first step, initialize the selected seed points, using a gradient search method to find out from the seed point closest to the surface point, which leads continuous expansion to the formation of the grid until it came to other seed points or formed their own grid; The second step, the tetrahedral grid is used to pick up the more uniform sampling points, and it can specify the resolution parameters to control the number of triangles; The third step, the grid optimization, can make a more uniform triangle. The third step, optimize the grid, can make a triangle more evenly. Algorithm operation step is as follows:First import point cloud data to calculate the normal vector at each point and unitization, then select and determine the impact of the center of the radius, by judgment until all the data points processed, solving its interpolation factor $\alpha$ and then the implicit surface mesh extract output reconstruction results. So get a good accurate registration effect.

\section{Summary}

This paper mainly introduces a new study method and the new system of coaxial measurement of marine propulsion shafting researching, including acquisition of the image information, the structure light calibration and 3D scanning point cloud data processing. This system can provide a new method and new idea for the coaxial measurement of marine propulsion shafting. The following will research how to apply the 3D scanning technology to the measurement of the coaxial surface of marine propulsion shafting and on the precision measurement technology, properly identified and addressed the tail shaft, concentricity error of the intermediate shaft and the engine shaft, to ensure that the ship propulsion shafting quality, reduce repair costs and shorten repair cycle have very important significance. At the same time, with the development of 3D scanning technology, it can be predicted that it will become the main instrument for the detection of the concentricity of marine propulsion shafting.

\section{Acknowledgment}

The research is supported by the research project of quality supervision department of Zhejiang Province (20130393) and public technology research and social development project of Zhejiang Provincial Department (2014C33107) and science and technology project of the Zhoushan city (2013C31049). The authors would like to express a special thank for its support. 


\section{References}

[1] Xinglin Zhou. Study on Large Section Geometric Shape Vision Measurement system [D]. Tianjin University , 2006. In Chinese.

[2] Pingliang Jie. Research on binocular vision reconstruction System by the structured light [D]. Technology of Guangdong University , 2014. In Chinese.

[3] Tingyu Wang. Study of Method of Coaxiality Measurement for Marine Shafting Based on CMOS [J]. Shipbuilding of china, 2014, 53(3): 175-178.In Chinese.

[4] Dexue Bi, Fangtao Liu, Qiang Xue, et al. New structured light vision sensor field calibration approach based on laser intersection lines [J]. Chinese Journal of Scientific Instrument, 2009,30 (8): 1697-1701. 